\title{
LA SUBJETIVIDAD ARGENTINA ATRAVESADA POR EL EJERCICIO DE LOS DERECHOS HUMANOS
}

\author{
Elio Rodolfo Parisí \\ Nacional de San Luis, Argentina. \\ Adrian Manzi ${ }^{2}$ \\ Nacional de San Luis, Argentina
}

http://dx.doi.org/10.5209/rev_NOMA.2014.v44.n4.49293

\begin{abstract}
Resumen.- La producción de conocimientos por parte de los organismos de derechos humanos de la Argentina que se suma al saber hacer, al saber resistir a las fuerzas de dominación mediante movilización constante y masiva más la denuncia pública, la consideramos de enorme magnitud y con un sentido que va más allá de la defensa y promoción de los mismos, ya que este saber hacer y este conocimiento otorgan la posibilidad de subjetivar a los ciudadanos como sujetos de derechos fundamentales. Nosotros abordamos el campo de los derechos humanos para analizar los mecanismos subjetivos que el ser argentino aplica a los otros y a sí mismo, para gobernarse. Es decir, cuáles son las estrategias y técnicas/tecnologías dentro de la gubernamentalidad que los ciudadanos argentinos utilizamos y que nos permiten distinguir y describir el uso reflexivo que hacemos de nuestra moderna libertad.
\end{abstract}

Palabras claves.- Derechos Humanos, Gubernamentalidad, Subjetividad, ONGs.

Abstract.- The production of knowledge on the part of the human rights organizations in Argentina that joins the know-how, the know how to resist the forces of domination through constant mobilization and mass public denunciation, we feel it is a huge magnitude and with a meaning that goes beyond the defense and promotion of human rights, since this "know-how and this knowledge granted the possibility of subjetivar citizens as subjects of fundamental rights. We are dealing with the field of human rights to discuss mechanisms subjective that the argentine identity applies to others and to himself, to govern themselves. That is to say, what are the strategies and techniques/technologies within the governmentality that Argentine citizens we use and which allow us to distinguish and describe the use reflective of our modern freedom.

Keys words.- Human Rights, Governmentality, Subjectivity, NGOs.

\footnotetext{
${ }^{1}$ Dr. En Psicología. Director Proyecto de Investigación Psicología Política. Universidad Nacional de San Luis, Argentina.

${ }^{2}$ Dr. En Psicología. Integrante Proyecto de Investigación Psicología Política. Universidad Nacional de San Luis, Argentina
} 


\section{Organismos de DDHH y análisis conceptual.}

La producción de conocimientos por parte de los organismos de derechos humanos de la Argentina que se suma al saber hacer, al saber resistir a las fuerzas de dominación mediante movilización constante y masiva más la denuncia pública, la consideramos de enorme magnitud y con un sentido que va más allá de la defensa y promoción de los mismos, ya que este saber hacer y este conocimiento otorgan la posibilidad de subjetivar a los ciudadanos como sujetos de derechos fundamentales. Logrando como resultado, quizás más sobresaliente, la judicialización de los delitos de lesa humanidad ocurridos durante el periodo de la última dictadura entre 1976 y 1982; también el desvelamiento de los índices y niveles de violencia de género y familiar, el aumento de aprehensiones y detenciones de los sectores más empobrecidos y el hacinamiento en cárceles y penitenciarías de los mismos, y la persecución de las redes de trata de personas con fines de explotación sexual, por nombrar lo más sobresaliente.

Entre los organismos más fundamentales podemos nombrar a: Madres de Plaza de Mayo, Abuelas de Plaza de Mayo, Centros de Estudios Legales y Sociales, Servicio de Paz y Justicia, Asamblea Permanente por los Derechos Humanos, Liga Argentina por los Derechos del Hombre, Movimiento Ecuménico por los Derechos Humanos, Equipo Argentino de Antropología Forense, Asociación Ex Detenidos Desaparecidos, Asociación Argentina de Prevención de Violencia Familiar, Indeso Mujer, Unión de Mujeres de la Argentina, Asociación de Mujeres Jueces en Argentina, Fundación Agenda de las Mujeres, H.I.J.O.S., Equipo Latinoamericano de Justicia, Comité de América Latina y el Caribe para la Defensa de los Derechos de la Mujer, Instituto de Género, Derechos y Desarrollo, Comité de Acción Jurídica, como los más importantes.

Así como Foucault abordó la sexualidad para analizar las diferentes formas de moral que el hombre se da a sí mismo cuando la conducta sexual se toma como entidad por pensar, o cuando abordó la locura para describir las formas de racionalidad que el hombre construye para dirimir el campo de lo normal con lo anormal, la razón de la locura; nosotros abordamos el campo de los derechos humanos no para describir al ser argentino en términos de qué tanto goza o en cuánta medida son vulnerados sus derechos fundamentales, sino para analizar los mecanismos subjetivos que el ser argentino aplica a los otros y a sí mismo, para gobernarse. Es decir, cuáles son las estrategias y técnicas/tecnologías dentro de la gubernamentalidad que los ciudadanos argentinos utilizamos y que nos permiten distinguir y describir el uso reflexivo que hacemos de nuestra moderna libertad.

El saber hacer y el conocimiento acerca de los derechos humanos acumulado durante los últimos 50 años en Argentina, disciplina a los individuos, gubernamentaliza a la población, como así también refiere a los modos de constitución de los sujetos, es decir, las prácticas, técnicas o mecanismos que respectan a los procesos de subjetivación de los argentinos. Creemos que cuando Foucault afirma que: "Pues bien, entre esas dos posibilidades, entre esos dos temas (el de una historia de las mentalidades y el de una historia de las representaciones), lo que procuré hacer es una historia del pensamiento. $Y$ al hablar de pensamiento hacía alusión a un análisis de lo que podríamos llamar focos de experiencia, donde se articulan unos con otros: primero, las formas de un saber posible; segundo, las matrices de comportamientos para los individuos; y por último, modos de existencia 
virtuales para sujetos posibles. Formas de saber, matriz de comportamientos, constitución de modos de ser del sujeto" (Foucault, 1983a); nosotros nos aproximamos, en la indagación de los derechos humanos, cómo un dispositivo nos permite pasar del análisis de la gubernamentalidad hacia los modos de constituirse como sujetos en un contexto determinado. Los derechos humanos en la Argentina configuran un foco de experiencia en el que se han configurado numerosos y múltiples sentidos en los ciudadanos.

\section{Introducción a la libertad argentina.}

Para nosotros, nuestras libertades fundamentales se dirimen, en gran parte, en el campo de la acción de la justicia. Esto es aceptar el hecho de que en la mayoría de los casos de los ciudadanos argentinos se están proclives a constituirse con las características de la estructura y forma de todo lo que implica, promueve y reproduce el sistema de derechos, y más precisamente, el sistema judicial.

Foucault propuso una metodología de abordaje al sujeto moderno y es ésta la que hemos ido desarrollando; a saber: "En vez de referirme a una teoría del sujeto, me pareció que había que procurar analizar las diferentes formas mediante las cuales el individuo se ve en la necesidad del constituirse como sujeto. $Y$, con el ejemplo del comportamiento sexual, traté de ver cómo y a través de qué formas concretas de relación consigo, el individuo se había visto ante la experiencia de constituirse como sujeto moral de su conducta sexual. Pasar de la cuestión del sujeto al análisis de las formas de subjetivación, y analizar esas formas de subjetivación a través de técnicas/tecnologías de la relación consigo mismo o, si lo prefieren, de lo que puede denominarse pragmática de sí" (Foucault, 1983a). Por lo que podríamos afirmar que, los mecanismos de relación entre los diversos sectores de la elegida experiencia Argentina en cuestión, constituyen técnicas o tecnologías de la relación con uno mismo y también de relación con los demás, si integramos el análisis de la anátomo política del poder, es decir, desde el disciplinamiento de la población argentina y del uso de la estadística como instrumento de la gubernamentabilidad. No intentamos ir hacia una teoría del sujeto a través del análisis de los casos que nos brinda la jurisprudencia nacional e internacional, o de adjetivar las conductas que llevan las formas de organizaciones no gubernamentales, es decir, sustancializar al sujeto argentino en términos de derecho, o moralizarlo según su dedicación solidaria. Más bien, hemos intentado caracterizar los procesos de subjetivación que se han ido conformando y consolidando a lo largo de la última época en la Argentina.

Si bien la tasa de desocupación, de la pobreza e indigencia, según los últimos datos del INDEC $^{3}$ la criminalización de la protesta social, la no represión a ciertas manifestaciones sectoriales han ido decreciendo, por un lado; por otro, el aumento de la población carcelaria y de las muertes de los presos, los altos índices de prostitución y la trata de mujeres y niños, la situación de los psiquiátricos ${ }^{4}$ y las condiciones de los internados y detenidos, la violencia de género, la violencia de miembros de las fuerzas de seguridad en hechos aislados, los procesos por los crímenes de lesa humanidad, los enjuiciamientos y sentencias a funcionarios policiales, las políticas de desalojo, los planes sociales y los beneficiarios, han ido en aumento. Todo esto configura el disciplinamiento argentino de su Estado y de su

\footnotetext{
${ }^{3}$ Instituto Nacional de Estadística y Censos.

${ }^{4}$ Salvo experiencias puntuales en la CABA y en Río Negro.
} 
sociedad. Lo fundamental es que existe un conocimiento que circula gracias a la voluntad de una sociedad civil que agrupa y reconstruye un tejido social, y a una política de gobierno que permite y alienta a que los distintos grupos sociales tengan voz e incidencia en la vida pública. Esta emergencia y amplia puesta en circulación de conocimientos, al menos en algunas provincias del país, habla también de la puesta en la agenda política, por parte del gobierno nacional, sobre algunos de estos conocimientos que se refieren a la violación de los derechos de manera sistemática en la Argentina.

Este conocimiento determina que la gubernamentalidad en la Argentina, si se toma como foco de experiencia a los derechos humanos, tiene características de expropiación de recursos humanos (30.000 detenidos desaparecidos), materiales (deuda externa y desindustrialización) y simbólicos (todo lo estatal es corrupto e ineficiente), con cierto marco de legalidad en términos legislativos y de normalidad en términos estadísticos.

Apuntamos al estilo de ética de la negación por parte de los funcionarios políticos, militares y civiles que se obstinan en no reconocer los delitos y a sus responsables. También enfocamos el silencio y la complicidad de gran parte de la sociedad que colabora con la no denuncia de todo tipo de delito que significa vulneración a los derechos más fundamentales y que deberíamos erradicar si se sigue una política de derechos humanos. Además destacamos lo sublime de las conductas de denuncia pública y movilización permanente. Pero evaluamos todos a estos mecanismos como productores de comportamientos, hábitos y subjetividades eficaces para el funcionamiento de la gubernamentalidad argentina.

Para nosotros no es sólo la impunidad a la que hay que perseguir y erradicar o a la corrupción y a la violencia en sí, cuestión que son objetivos que van de suyo, sino el hecho de evaluar cómo y qué podemos hacer con estas modalidades de funcionamiento, estos modos de ser que ya están instalados. No vislumbramos contradicciones en estos modos de funcionamiento subjetivo de los argentinos (unos versus los otros); sino la posibilidad para cualquiera que habite el suelo argentino de ejercer la relación con los demás y consigo mismo, de aplicar cualquiera de estos modos de subjetivación.

Los procesos de subjetivación abren la posibilidad de negar, callar, denunciar, ser cómplice, movilizarse, desvalorizar, solidarizar, culpabilizarse, financiar, olvidar, memorizar, indultar, entre nosotros. No sólo son momentos en la conducta, también tienen su correlato en las instituciones públicas. Tenemos conciencia que no son los únicos modos por los cuales, dentro de la experiencia de los derechos humanos, existe la posibilidad de constituirse como sujetos. Hay otros cursos o modalidades de subjetivación, que no necesariamente implican solamente a estos mecanismos e involucran a todos los demás argentinos. Aún así, constituye como objetivo importantísimo la erradicación o disminución de la pobreza, la delincuencia, la prostitución, la impunidad, la violencia de las instituciones de seguridad, en la familia, y en comercios de noches; en los asilos, en los hospitales; como así también resulta necesario enfocar y reflexionar sobre la forma ética ya instalada en los argentinos de negar, silenciar, denunciar, movilizar, culpabilizar, desvalorizar, solidarizar, enjuiciar, procesar, imputar, procesar, financiar, indultar.

Alfredo Grande (2002), en su "psicoanálisis implicado" afirma que en la actualidad hay un modo yoico de producción de subjetividad el cual mantiene unido a la Realidad con el Deseo en una relación no contradictoria, es decir, una unión 
profundamente ética. La lógica del deseo, dice él, organiza los modos yoicos de producción de subjetividad. Lógica que se ordena según la tensión entre el Yo y el Ideal del Yo, denominada sentimiento de autoestima, la cual permite al sujeto, cuando la distancia de éstas es considerable, tolerarse y amarse sin tener que pedir perdón. Es decir, que la cercanía entre el Yo y el Ideal del Yo permite cierta alegría por el deseo, pues responde más a un fundamento deseante que es proclive a la descarga; y no a una alegría por el mandato que en realidad es manía, cuando la brecha es grande, que es proclive a la contra carga. La primera alegría, responde a una gloria "hacer lo que se quiere" y la segunda, a una mediocre resignación de "querer lo que se hace". Con la aclaración de que hacer lo que se quiere no es hacer cualquier cosa: es fiesta, no carnaval, dice Grande. El modo yoico que permite la esperanza, los proyectos, los anhelos, es aquel que establece alianzas fraternas y que prolonga las demandas del Ello, para el cual la Realidad no es ni sometida ni dominadora. La lógica yoica produce creencias, y la lógica superyoica (aquella que funciona más con los mandatos del Ideal del Yo) produce certezas, ya que su génesis está en la amenaza de castración. Castración que una vez fue consolidada a través del paso por el complejo de Edipo y su declinación. Su eficacia tiene que ver no con el anuncio de un castigo, sino con castigar con la manipulación de la amenaza (función de la contra carga). Ésta es el castigo mismo ya que es fundadora de la estructura deseante: la declinación del deseo -incestuoso-por la madre y el padre, estructuran nuestra mente en tanto sujetos deseantes, a partir de lo que una vez y para siempre fue prohibido y perdido, pero no por eso abandonado y olvidado.

De aquí que Grande puede afirmar que: "los desocupados han logrado subvertir el fundamento culpógeno que somete a los sujetos a los diversos sistemas de dominación que la sociedad capitalista organiza. Quizás la metapsicología del piquete tenga que ver con la consistencia, coherencia y credibilidad de las alianzas fundantes de la vida que vuelven a ser el motor de las historias. Los porcentajes de los desocupados operan como amenaza de desocupación sobre los que aún tienen cierto grado de narcisismo laboral. El sujeto no se constituye como 'ser en falta', sino como 'existente de la amenaza'. El modo superyoico de producción de subjetividad captura toda la producción deseante. El objeto del deseo pasará a ser oscuro, peligroso, contagioso, escaso" (Grande, op. cit.).

$\mathrm{Si}$ el objeto del deseo en esta cultura de mercado lo constituye el trabajo, como tantos otros objetos deseables, la carga sobre éste con base de una identificación más estructurante con el Ideal del Yo (modo superyoico de subjetividad), produce sentimientos de culpa, de castigo, de renuncia, de amenaza, de sacrificios. El Ideal del Yo transforma en su contrario el plus de energía destinado a su objeto catectizado, es decir, que la contra carga recae en el yo cuando el trabajo (o el salario) sufre la prohibición como objeto del deseo. $\mathrm{Y}$ si el trabajo es un objeto escaso, el desearlo tiene su precio. "Freud dice que cuando el Estado se opone a la violencia no es para prohibirla, sino para monopolizarla. El periodo de latencia es el proceso de institucionalización del Estado en nuestra subjetividad inconsciente. ¿Qué mejor represión que la que nosotros mismos ejercemos sobre nosotros mismos? Armamos nuestras propias fuerzas de seguridad intra psíquicas que nos previenen de los enemigos exteriores e interiores. Aquellos que no podrán provocar nuestros deseos" (Grande, op. Cit.).

Lo que significa que en la cultura represora y de la ley (no de la ley legal, sino de la ley que estructura el modo de producción yoica), y no de la represión y de la prohibición; se requieren mecanismos que aseguren comportamientos que no sólo 
repriman el deseo, sino en los que se desee represión. Así se ha estructurado nuestra lógica del deseo. Represión, secuestro, muerte, negación, investigación frustrada, denuncia, movilización, negación, complicidad, solidaridad, procesos, indultos, penas. "Cuando el sujeto adquiere la más completa convicción de que la única forma de vivir es morir un poco todos los días, el Super Yo (hijo de la declinación del Edipo, estructura consolidada a partir de la identificación con el Ideal del Yo) comienza a disfrutar de su triunfo. Es la crónica de una muerte anunciada. Anunciada por los ideólogos de los sistemas de dominación que nos hablan de los desocupados, mercados emergentes, reconversión de la economía, chicos de la calle, riesgo país, costos social del ajuste. Formas encubiertas de hablar de los genocidios de baja y mediana intensidad" (Grande, op. Cit.).

Por lo que entendemos que la unión entre la Realidad y el Deseo no es contradictoria en sí (que la primera se impone a la segunda, o que ambas otorgan diferentes sentidos a la vida); sino que es productora de modos de funcionamiento psíquico que permiten mantener la tensión entre el yo que desea y el yo que obedece. La tensión yoica (producción de subjetividad), no está entre la ley y legalidad, sino entre el impulso y la norma, entre la dignidad y la represión. No es que unos mecanismos y deseos van a parar al inconsciente y otros son conscientes; más bien son todos mecanismos inconscientes que aseguran la estabilidad del yo debido a la tensión fundante entre el objeto del deseo (oscuro, escaso, difuso) y lo que la realidad dictamina como posible y permitido para desear (con culpa, con desvalorización, con amenaza), en este ejemplo; el trabajo.

No observamos a estos mecanismos subjetivos y movimientos de la población argentina como síntomas de sólo un trauma social vivido años atrás, sino más bien como potenciadores y productores de una particular manera de gobernarse a uno mismo y a los otros, caracterizada como perversa, cuando la libertad es practicada. No son dinámicas sobre las cuales hay un sentido que interpretar, sino modos de funcionamiento sobre los cuales hay que intervenir. No es el malestar en la cultura por diagnosticar, sino el establecer qué se está haciendo mientras tenemos conocimiento de los índices del tipo de administración de la vida, de los estilos de muertes que generamos, los desocupados que mantenemos, las niñas y niñas que perdemos.

Sostenemos que los derechos humanos constituyen un dispositivo de seguridad de la gubernamentalidad, según los desarrollos conceptuales de Foucault durante los años 1978, 1978 y 1979 en el Colegio de Francia. Y también, que la presencia de agencias operativas claves, como la civil (todas las organizaciones sin fines de lucro, sobre todo las llamadas ONGs) y la judicial (todo el sistema judicial), constituyen a la economía política de la población argentina cuando ésta hace uso de la moderna libertad. También afirmamos que la desocupación, la precarización del trabajo, la criminalización de la pobreza y de la inmigración, la judicialización de la protesta social, el abuso infantil, la violencia de género, la violencia policial y militar institucional y los pactos políticos con sectores financieros, son la estrategia política de los sectores perversos que ejercen el poder, imponiendo la fuerza material y simbólica, expropiando recursos humanos y materiales.

En la Argentina, la experiencia de la detención y desaparición de miles de personas, a través de un plan sistemático organizado por quienes detentaron el poder estatal durante la dictadura, fue investigada en el "Informe Prohibido" de 1979 de la Comisión Interamericana de DDHH cuando realiza la visita "In locus" a la Argentina 
en ese año, y en 1984 por la Comisión Nacional de Desaparición De Personas da a conocer su "Nunca Más". Y la experiencia de expropiación del casi total de sus recursos humanos, materiales y simbólicos también durante las décadas siguientes, por medio de la complicidad civil y de los funcionarios públicos, que se comprueba con la crisis de diciembre de 2001. Esto hace que enfoquemos a los derechos humanos como entidad no muy bien definida en cuanto constituyen una problematización para la economía y la política actual; como lo muestra la difusa preocupación de la sociedad en general por "la seguridad" de los ciudadanos, o por el clientelismo que se establecen desde los planes sociales. Y, además, los derechos humanos ofrecen la posibilidad de diagnosticar la condición que hay para los argentinos en hacer uso de las instituciones y valores culturales. El aumento de la judicialización de muchos de los aspectos sociales de la Argentina, se ha hecho una práctica común, tendencia política homogeneizante, del ejercicio del poder, en términos de la dominación en nuestro territorio, de nuestros recursos y de nuestras prácticas sociales.

Como bien dice Foucault (1978), "todas las grandes máquinas disciplinarias (cuarteles, escuelas, talleres, y prisiones) son máquinas que permiten cercar al individuo, saber lo que es, lo que hace, lo que puede hacer, dónde es necesario situarlo, cómo situarlo entre otros. La importancia de la estadística es que permite medir cuantitativamente los efectos de masa de los comportamientos individuales".

A partir del análisis del conocimiento producido en materia de derechos humanos, ha sido posible determinar la maquinaria disciplinante argentina, y caracterizar a los mecanismos de racionalización económica y estabilidad política que tienen, tanto efectos individualizantes, como de masa, es decir su racionalidad gubernamental.

Las estadísticas de los informes publicados por parte de los diversos organismos de derechos humanos permiten, a nuestro entender, el análisis cualitativo de las relaciones de poder. Los conocimientos producidos por los mismos tienen alcances inigualables a nivel social, político y jurídico internacional. Además, no sólo los consideramos como material que caracteriza al disciplinamiento del individuo a nivel corporal y que indica la gubernamentabilidad de la población, sino también, como amplificador de nuestros modos de constituirnos como sujetos.

\section{Conocimientos de Derechos Humanos.}

EI INDEC (Instituto Nacional de Estadística y Censos) en su página oficial ${ }^{5}$ tiene publicado los índices de empleo y de desocupación, entre otros. Por un lado, no es casual que muchas de las publicaciones tengan datos que comienzan desde el año 2003, inicio del gobierno del ex presidente de la nación Néstor Kirchner, y que la actual presidenta Cristina Fernández de Kirchner continúa con el plan político. La cuestión es que se quiere resaltar una diferencia de estos gobiernos con los anteriores. Por otro lado, tampoco es casual que la tasa de desempleo haya descendido, ya que en conjunto han estado aplicando medidas que de alguna manera la Argentina que hemos venido describiendo y analizando, las necesita. Al primer trimestre del 2003 la tasa de desocupación de los conglomerados urbanos (aquellos de más de 500 mil habitantes) fue de 20,4\%, al primer trimestre del 2004 fue de 14,4\%, al primer trimestre del 2005 fue de 13,0\%, al primer trimestre del 2006 fue de 11,4\%, al primer trimestre del 2007 fue de 9,8\%, al primer trimestre del 2008 y

\footnotetext{
${ }^{5} \mathrm{http}: / /$ www.indec.mecon.ar/nuevaweb/cuadros/4/EPH_cont_4trim10.pdf
} 
del 2009 fue de 8,4\% y al primer trimestre del 2010 fue de 8,3\% llegando al 7,3\% al último trimestre de 2011. El porcentaje de los aglomerados del interior, sin contar con Gran Buenos Aires, rondan estadísticas similares. Van desde el $18,8 \%$ de desocupación en el 2003; pasando por $14,1 \%$ al $2004 ; 11,1 \%$ al $2005 ; 10,0 \%$ al $2006 ; 8,3 \%$ al 2007; 7,7\% al 2008; 8,3\% al 2009; 8,1\% al primer trimestre del 2010 finalizando en 6,4 en este año.

Hay otras publicaciones que se guían por otras fuentes, cual puede ser el de la C.I.A. World Factbook. ${ }^{6}$ Si bien los índices no son exactamente los mismos, es reconocible el notable descenso de la tasa de desempleo. Indica, esta fuente, un $21,5 \%$ de desempleo al 2003 , un $17,3 \%$ al 2004 , un $14,8 \%$ al 2005 , un $11,6 \%$ al 2006 , un $8,7 \%$ al 2007 y un $8,9 \%$ al 2008. Las políticas públicas que están llevando a cabo los gobiernos de los Kirchner, implicarían la posición activa del Estado frente a determinados sectores sociales. No sólo frente a los más desamparados o abandonados, sino también frente a algunos grupos con cierto poder. El interés por disminuir la desocupación y generar empleo ha sido una política seria, ya que con ella no sólo se ha logrado un crecimiento económico en general (que no quiere decir bien distribuido), sino que también se ha ido avanzando en generar trabajo en blanco y en perseguir el trabajo esclavo. No se puede dejar de reconocer que, en esta Argentina del silencio y la complicidad, estos gobiernos están prestando oídos a ciertos reclamos que se escuchan, sobre todo, desde los organismos no gubernamentales, como lo son los de defensa por los derecho humanos.

Por su parte, el campo de conocimientos sobre el que se desplazó el accionar de los organismos de derechos humanos, es vasto, complejo y polimorfo. No sólo es el sentido y significado que otorgaron a sus accionares y a sus conocimientos, sino, a los ámbitos de aplicación sobre los cuales ejercen su poder.

Las intervenciones, o al menos intentos de injerencia e influencia a nivel político que podemos nombrar, a modo de ejemplo: haber llevado a cabo los juicios por crímenes de lesa humanidad; y como describiéramos anteriormente, la preocupación por la localización y restitución a las legítimas familias de todos los niños secuestradosdesaparecidos, ha sido un objetivo más que primordial. El número de imputados es de 1422 hasta diciembre del 2009, de los cuales 1179 están en condiciones de enfrentar juicio y los restantes 243 fallecieron o fueron declarados incapaces. Los procesados llegan a 628, los cuales 410 se encuentran cumpliendo prisión preventiva, más 50 sentenciados (47 condenados y 3 absueltos). Los prófugos se mantienen en 40. (CELS, 2009) Para muchos el 2010 fue el año de mayor importancia para los juicios por estos tipos de delitos. Se dictaron 107 condenas a represores, la mayor parte a Prisión Perpetua o a 25 años de prisión, en juicios celebrados en la Ciudad de Buenos Aires y en las provincias de Córdoba, Santa Fe, Tucumán, Santiago del Estero, Buenos Aires, Chaco, La Rioja, La Pampa; Mendoza y Chaco. Solo en las provincias de San Juan, Jujuy, Catamarca, Entre Ríos, Santa Cruz, Chubut, Río Negro y Ushuaia no hubo condenas. (CELS, 2010) En cuanto a los niños restituidos de su identidad, aquellos que fueron nacidos en cautiverio y otorgados a familiar ilegalmente ya suman los 104. (Abuelas de Plaza de Mayo 2010).

Existen además intervenciones y enfoques de reformas democráticas en las Fuerzas Armadas; sobre el papel que juega la asistencia religiosa en las mismas; debates

\footnotetext{
${ }^{6}$ http://www.indexmundi.com/es/argentina/tasa_de_desempleo.html
} 
sobre el Código de Justicia Militar, investigaciones sobre los niveles de violencia militar, en el ámbito federal y provinciales de la policía (CELS, 2009). De esta manera, la preocupación por las políticas de seguridad pública, tanto a nivel de debate social como a nivel de análisis político socioeconómico ha sido, y es, materia importante para la agenda pública, según alegan los organismos de derechos humanos. Dentro de esta temática, la preocupación por la represión y criminalización de las protestas sociales ha dado por resultado que el Estado haga oídos y opte por una política no represiva (CAJ, 2006; FIDH, 2006).

Pero, tanto para el CELS como para el CAJ (Comité de Acción Jurídica), el CEJIL (Centro por Justicia y el Derechos Internacional), la LADH (Liga Argentina por los Derechos Humanos), la CORREPI (Coordinadora Contra la Represión Policial e Institucional) $^{7}$ y la FIDH (Federación Internacional de Derechos Humanos), estos organismos concluyen que el poder estatal lejos de utilizar el sistema penal como recurso extraordinario, lo utiliza como mecanismo de control social. Según el CELS (2003) en el 2002, la CTA (Central de Trabajadores Argentinos) y el CAJ, elevaron una queja al Comité de Libertad Sindical del Consejo de Administración de la OIT (Organización Internacional del Trabajo) en la que se denunció que más de 2.800.000 personas, empleadas o desempleadas, se encontraban bajo proceso penal por incidentes sindicalizados; y un año antes la presentación había sido ante la $\mathrm{CIDH}^{8}$ en conjunto con CEJIL. En varias presentaciones y audiencias con la CIDH, durante el 2001, se denunció la conducta violatoria del Estado argentino contra los derechos humanos. "El $70 \%$ de los procesos vinculados con la protesta social se refiere a la modalidad conocida como 'corte de calles o rutas', es decir, al delito de entorpecimiento del tránsito" (CAJ, 2006).

La situación de los penitenciarios de las cárceles constituye otro objetivo fuerte en las agendas de los organismos de derechos humanos. Así como también los temas de superpoblación carcelaria, falta de higiene y de políticas de salud sobre los enfermos encarcelados, investigación sobre casos de tormentos y torturas, sobre los mecanismos de detención y hacinamiento en penitenciarías, y monitoreo sobre los lugares de detención. Según el CELS (2005) en la Argentina más de 63.000 personas se encuentran privadas de su libertad en establecimientos carcelarios y dice que: "La Argentina ha optado por la utilización del derecho penal como principal método de solución de conflictos y el empleo de la prisión como pilar del sistema punitivo". En la provincia de Buenos Aires, la cantidad de aprehensiones realizadas por la policía se incrementó, entre 1990 y 2007, más del 150\%, es decir, de 71 personas privadas de libertad cada 100.000 habitantes en 1990 a 181 en el 2007 (CELS, 2010). Actualmente, en la provincia de Buenos Aires permanecen alojadas casi 30.000 personas privadas de libertad en condiciones degradantes, sometidas a situaciones de violencia sistemática (CELS, 2010). En la provincia de Buenos Aires las aprehensiones por delitos han tenido un constante incremento. El 2002 tuvo 81.012 aprehensiones, el 2003 tuvo 88.140 aprehensiones, el 2004 tuvo 112.349 aprehensiones, el 2005 tuvo 153.472 aprehensiones, el 2006 tuvo 153.472 aprehensiones, el 2007 tuvo 129.340 aprehensiones, el 2008 tuvo 202.596 aprehensiones, el 2009248.546 aprehensiones. Hasta hace pocos años "se verifica la continuidad de las prácticas de detención de personas sin revisión judicial y

\footnotetext{
7 Ver "Informe de situación 2003-2006: Los Derechos Humanos en la Gestión Kirchner". http://www.rebelion.org/noticia.php?id=36784

${ }^{8}$ Comisión Interamericana de Derechos Humanos.
} 
vigencia, en los códigos contravencionales de varias provincias" (CELS, 2009). Entre 2002 y 2006 la cantidad de menores detenidos se triplicó (CELS, 2009). El relevamiento realizado por la Secretaría del Ministerio de Justicia y Derechos Humanos de la Nación reveló que unos 20.000 niños y adolescentes están privados de su libertad, de los cuales el $87,1 \%$ está internado por situación socioeconómica y sólo el 12,1\% por causas penales (CELS, 2010)

La preocupación por lo social, como lo son los estudios sobre déficit poblacional y los desalojos sobre la base de políticas de expulsión, más que nada en la provincia de Buenos Aires, como así también los estudios sobre los niveles de calidad de vida de sectores populares, tienen sus presentaciones ante la Corte Suprema de Justicia de la Nación por parte de los organismos citados.

Los seguimientos de control en materia de cumplimiento de entrega de planes sociales por parte del Estado Nacional o de los Estados Provinciales, son correlativos al monitoreo sobre el impacto real y concreto de los planes sociales, como así también a los análisis sobre distribución de ingresos y de las propuestas de recomposición salarial, régimen de asignaciones familiares, salario mínimo, vital y móvil. Aún cuando el gobierno actual reciba críticas precisas por parte de los organismos de derechos humanos, y desmedidas por parte de la oposición, sobre la aplicación de la "Asignación Universal por Hijo", no deja de imprimirle esta política de Estado un rol no meramente subsidiario; ya que tiene por objetivo, esta asignación, facilitar el reingreso de los niños del sector pobre a las escuelas, y el ejercicio de los controles sobre la salud, ya que los padres deben presentar el calendario de las vacunaciones completo y su libreta escolar.

Dentro del ámbito social, la injerencia de los organismos, a nivel del conocimiento se encuentran protagonizados por realizaciones de coloquios interdisciplinarios en identidad y memoria, congresos de economía política, en materia de salud mental, y seminarios de participación en debates de intelectuales y profesionales nacionales y extranjeros. La creación de la Universidad de Madres de Plaza de Mayo y de toda su línea editorial ha contribuido enormemente al bagaje de la producción de conocimientos en Ciencias Humanas y también Económicas. (Madres de Plaza de Mayo, 2005, 2006, 2007; Abuelas de Plaza de Mayo, 2002, 2003).

La situación de los indígenas se enfoca sobre la asociación y participación política de los mismos y sobre sus reclamos territoriales, además de las denuncias sobre persecución política y policial a los mismos (CELS, 2010; SERPAJ).

También son patrocinadores en conjunto con otros organismos internacionales de aquellos que necesitan ejercer su derecho a la defensa y que se encuentran en los servicios de salud mental del país, por sus situaciones de hacinamientos, institucionalización, medicalización, sobrepoblación, precarización, defunciones. Como lo investigaron el CELS -en conjunto con el Mental Disability Rights International (MDRI)- durante junio y diciembre de 2004, junio de 2005, enero y setiembre de 2006, se documentaron 25.000 personas segregadas en asilos, y en psiquiátricos argentinos, a lo que se las denomina personas institucionalizadas, internadas, o detenidas. Más de un $80 \%$ de estas personas son encerradas durante más de un año, y muchas de por vida: "Las autoridades admiten que entre el $60 \%$ y el $90 \%$ de las personas detenidas en las instituciones son 'pacientes sociales', nombre que los funcionarios y profesionales les dan a las personas que no tienen familias que las contengan y permanecen internadas como consecuencia de la falta de recursos de la comunidad" (CELS, 2008a) El mismo organismo, en su informe del 
2009, dice que, durante el 2007 y el 2008, se iniciaron más de 16.152 procesos judiciales por cuestiones de salud mental, entre las cuales se citan: internaciones forzosas, control de internaciones inicialmente voluntarias, insanias e inhabilitaciones; de las cuales sólo 256 casos tuvieron sentencia o resolución definitiva.

La preocupación por los temas de aplicación en políticas públicas sobre el ejercicio a la libertad de expresión y sobre la televisación de los juicios por violaciones masivas a los derechos humanos, es decir, de acceso a la información, también tienen su lógica de correspondencia con las propuestas de debates públicos sobre temas de la despenalización del aborto y sobre la situación discriminatoria de las mujeres en general.

Sobre esto último, los temas específicos sobre la cuestión tratan de: la participación de la mujer en cargos públicos y la falta de equidad en el ámbito laboral con respecto a los hombres, sus derechos en el ámbito de la salud, las posibilidades que tienen de educación sobre la reproducción sexual y de educación misma, los problemas sobre identidad de género y de diversos géneros, su situación dentro de la familia y la violencia que en ellas se viven, y también el reconocimiento de la plurinacionalidad y el multiculturalismo, explotación de la prostitución, trata y tráficos de mujeres y niños. Respectivamente, "desde el punto de vista del derecho, la violencia ha sido asociada al delito y, luego, se la consideró un conflicto de tipo familiar o social. En 2004, hasta octubre, se habían presentado 2.621 denuncias en la causa civil; de ellas, 2.471 habian tenido intervención policial previa" (ELA, 2005). EI ELA considera que existe una subestimación sobre este fenómeno impidiendo enfocarlo como corresponde y, que a la vez, es innegable la relación entre este tipo de violencia y la violencia de género.

En cuanto a la violencia contra la integridad sexual y el honor, el informe del Sistema Nacional de Información Criminal (SNIC), dice que en el 2003 se registraron 10.255 delitos contra la integridad sexual y el honor en todo el país. De éstos, 324 son delitos contra el honor, 3.448 son violaciones, 6.483 son otros delitos contra la integridad sexual.

Tanto la Organización Internacional para las Migraciones (OIM), como la Comisión Interamericana de la Mujer de la OEA (CIM-OEA) tienen en claro que en la Argentina predomina la trata interna, aún cuando hayan sido identificados casos de trata internacional, sobre todo mujeres paraguayas y en menor medida: brasileras, dominicanas y colombianas. ${ }^{9}$ Se han detectado redes de prostitución infantil y turismo sexual con niños/as y adolescentes. El mapeo de la Red de Trata ${ }^{10}$ describe la relación existente entre la presencia de la violencia sistemática y las actividades de expropiación de personas para ofrecerlas al mercado. El contra informe presentado por un grupo de organizaciones no gubernamentales al Comité de la

\footnotetext{
${ }^{9}$ La Red contra la Trata de Personas, OIM (2008), CEIM-OEA (2002), CASADICN (2006), Obispado de Viedma, el CAV (Centro de atención a la Víctima, Comodoro Rivadavia, Santa Fe, 2006), CAREF (Comisión Argentina para los Refugiados, perteneciente a las Iglesias Evangélicas en la Argentina).

${ }^{10}$ Entre las instituciones que conforman la Red pueden mencionarse: Fundación Mujeres en Igualdad, CECYM (Centro de Encuentros, Cultura y Mujer), Defensoría de la Ciudad de Buenos Aires, Programa Todas de la Dirección de la Mujer del GCBA. Procuración General de la Nación, Colegio Público de Abogados, Consejo Nacional de la Niñez, Adolescencia y Familia, Consejo Nacional de la Mujer, Secretaría de Desarrollo Social del GCBA, Coalición contra el Tráfico de Mujeres, Foro de Derechos Reproductivos.
} 
CEDAW en el 2002, asienta que "la cantidad de sentencias condenatorias es diez veces menor a las denuncias. Se calcula que sólo se denuncian el $10 \%$ de los casos, por lo que habrá 60.000 casos de delitos sexuales al año, o sea 16 casos diarios." Según este mismo informe, todas las mujeres en situación de prostitución, han sido objeto de reiterados abusos, apremios y detenciones ilegales por parte de las fuerzas policiales.

Es de rescatar que el miembro del Estado Nacional ha acompañado, por medio de funcionarios o presencias institucionales, en muchas ocasiones a congresos y reuniones de carácter nacional e internacional en donde la temática de la mujer es la agenda principal.

La situación de los migrantes e inmigrantes ocupan temáticas tales como la reglamentación de las mismas, violaciones a la protección familiar, al arraigo del país, igualdad de derechos para nacionales y extranjeros, trabajadores migratorios, refugiados políticos, xenofobia.

En cuanto a los derechos laborales, investigan los sucesos de los diversos conflictos sindicales y son querellantes y patrocinadores en diversas causas. También han denunciado en el último año, a funcionarios públicos y empresarios, que mantienen bajo su mando a trabajadores en situaciones de trabajo en negro y hasta de casi esclavitud.

También vuelcan su atención sobre la situación, estructura y características sobre el Sistema de Justicia de la Nación, publicando informes y presentándolos ante la misma justicia nacional e internacional e intentando que los debates se vuelquen a la agenda pública, proponiendo enfoques y cambios sobre el Consejo de la Magistratura de la Nación, analizando las situaciones particulares de cada sistema de cada provincia.

A partir de entonces, creemos es imprescindible analizar la relación que existe entre los actuales índices de pobreza e indigencia que el Estado liberal mantiene y las respuestas estatales represivas frente a esta estadística. Consideramos, por un lado, que la libertad consiste a partir de tener posibilidad de elegir mientras se vive. $Y$ en ese límite difuso entre lo que se puede, lo que se quiere y lo que se sabe, se manifiestan los diferentes usos acerca de la misma en nuestra liberal contemporaneidad.

Un poco en contra de lo que señaláramos anteriormente con los índices vertidos por el INDEC, el CELS (2003) indica que en mayo de 2002 se produjo el récord en el índice de desempleo, llegando al $21,5 \%$ de la población económicamente activa, disminuyendo apenas para fin de año a partir de que 2 millones fuesen beneficiarios de planes sociales: "Los picos de desocupación se registraron en el Gran Catamarca (20,5\%), Gran Santa Fe (20,1\%), Jujuy - Palpalá (20,5\%) y en los partidos del conurbano bonaerense (21\%). En la década del 70 , el $5 \%$ de la población vivía en hogares con ingresos bajo la línea de la pobreza, en los 80 se elevó al 12\%; a partir de 1998 ya superaba el 30\% y en octubre de 2002 llegaba al 57,7\% (aproximadamente 20,7 millones de personas). A su vez, 9,9 millones se encuentran por debajo de la línea de indigencia (el 27,5\%) de la población." Ya para el 2008 casi 10 millones estaban en situación de pobreza e indigencia. Más del $40 \%$ de los menores de 14 años son pobres, y en las regiones como el noroeste la cifra llega al $60 \%$ (CELS, 2008). "Durante el primer semestre de 2009, se encontraban por debajo de la línea de la pobreza 709.000 hogares (9,4\%), los que incluyen a 3.429 .000 personas (13,9\%). De ese conjunto, 232.000 hogares $83,1 \%$ se encuentran bajo la 
línea de indigencia., lo que supone 995.000 personas indigentes. Según el INDEC la tasa de desocupación en el segundo trimestre de 2009 fue del 8,8\%. La desocupación entre los jefes de hogar fue del $5 \%$. La mayor tasa se observa entre las mujeres de hasta 29 años (18,6\%), mientras que la más baja se registra entre los varones de 30 a 64 años (5,2\%). El Observatorio de la Deuda Social Argentina revela que, en los conglomerados urbanos de 200 mil habitantes o más, el desempleo trepó del 10,1\% al 11,8\% entre 2008 y 2009. En el interior del país, el incremento pasó del 7,4\% en 2008 al 12\% en mayo de 2009" (CELS, 2010). Más allá de la discrepancia de los datos suministrados por este organismo de derechos humanos con los del Estado Nacional, o de la incredulidad que se tiene de los datos de esta institución, lo cierto es que existe una gran mayoría, de diversas clases sociales, con empleo estable, precario o sin empleo, en tanto que jóvenes, mujeres y adultos siguen volcando sus demandas ante al Estado.

\section{Subjetividad argentina de los derechos humanos.}

Ahora bien, en nuestro interés por destacar los modos de subjetivación, las técnicas o tecnologías para constituirse como sujetos, en tanto para con uno mismo y en tanto para los otros; se hace necesario realizar el desplazamiento del que más arriba citáramos. Ya que para Foucault, "la gubernamentalidad implica la relación de uno consigo mismo, lo que significa que en esta noción de gubernamentalidad, apunto al conjunto de prácticas mediante las cuales se pueden constituir, definir, organizar e instrumentalizar las estrategias que los individuos, en su libertad, pueden tener los unos respecto de los otros. La noción de gubernamentalidad permite, eso creo, hacer valer la libertad del sujeto y la relación con los otros, es decir, lo que constituye materia misma de la ética" (Foucault, 1984). De aquí que nuestra preocupación se vuelca hacia el análisis cualitativo de los conocimientos adquiridos, producidos y propuestos para la agenda pública y política, ya que éstos hablan, a nuestro entender, de la intensidad y las modalidades de las relaciones de las fuerzas del poder que aquí, en Argentina, se imponen, más que se distribuyen.

Comprendemos que, desde los espacios abiertos, se ha disciplinado mediante la criminalización de la protesta, podríamos decir, impunemente. Pero no es una política que actualmente esté continuando sin reparar en el pasado, sino que más bien intenta desde el Estado, lo contrario. A la luz de todos los medios, a la vista de toda la ciudadanía, la represión seguida de criminalización, fue la consecuencia de la naturalización de relación entre actores sociales por medio de la negación y el silencio; con impericia y en connivencia, es decir, con complicidad. Las actuaciones de represión, es decir, de respuesta estatal frente a movilizaciones sociales o de disciplinamiento de la sociedad excluida, lograron expresarse a cielo abierto en presencia de civiles y de periodistas. La violencia producida-ejercida y naturalizada que se ejerció desde el Estado hacia ciertos sectores de la sociedad, quedó impregnada en el imaginario de los demás actores sociales. Pero también ha quedado impregnado en el imaginario colectivo, que la violencia se ejerce también desde los grupos, que de manera masiva, ejercen su derecho al reclamo. La naturalización de la violencia se ejerce también, por ende, desde el desconocimiento de la información.

Para unos, es urgente crear nuevas políticas de seguridad sobre las ya naturalizadas políticas represivas, a base de una re-estructuración policial. Esto evidencia que aún existe una gran cantidad de sectores que, cautivas de los 
mecanismos de negación y silencio, de complicidad y desinformación, no logran distinguir cuáles son exactamente las causas de la violencia entre el Estado y los sectores protestantes. Creen que la policía es la instancia decisiva para el control del delito. Para otros, la propuesta de la seguridad ciudadana, tanto para los hechos delictivos aislados como para las protestas sociales y otros también, arranca desde el fortalecimiento de las demás instituciones democráticas (empezando por la escolaridad). Hay sectores del Estado Nacional que están escuchando actualmente las demandas de los grupos de derecho humanos, aún cuando éstos no abandonan la crítica ni la investigación de la realidad. De todas maneras, la presencia de estos modos de ser, en el que la violencia media en el ejercicio de la libertad (a manifestarse, a la huelga, a robar) entre los ciudadanos, ha largado cifras constantes y estables durante la década del '90 y la pasada.

No sólo es el sector pobre el que quedó incluido en la lógica de la cultura represora en este complejo proceder de la gubernamentalidad, es decir, los índices de violencia generalmente han incluido al sector más vulnerable, ya sea por víctimas o por victimarios. El sector de las fuerzas armadas y del poder judicial, funcionarios de justicia y actores civiles, también operaron, y operan todavía muchos de ellos, disciplinándose a su manera y con estos modos de ser entre los cuales: ante la movilización y la solidaridad, la denuncia pública y la judicial; se responde con más expropiación, más desvalorización, represión, negación de los hechos y complicidad de competencias por parte de los funcionarios de justicia. La violencia micro política, que recién está saliendo a la luz, da cuenta de los índices de complicidad que el poder represor necesitó y ejerció.

Ya sean jueces y fiscales, constitucionalistas y abogados, pasando por cabos primeros hasta carceleros de la policía y gendarmería, desempleados piqueteros y subempleados piqueteros, querellantes y testigos, docentes y científicos, empresarios y sindicalistas, no comparten las mismas condiciones materiales de existencia, el mismo devenir en el mercado, el mismo estatuto en el derecho, ni tampoco la misma ideología humanística, ni la misma clase social. Por lo que queremos acentuar que los modos de relacionarse entre estos sectores, -para caracterizar a la gubernamentalidad- están desplegados extensivamente y reticularmente en el tejido socio político argentino. Desde las altos estratos hasta los más ínfimos escalafones. Sin embargo, rescatamos que desde el actual gobierno nacional se intenta disminuir la actuación violenta y represiva por parte del Estado.

Para Pavlovsky (1999) "fueron la fuerzas armadas las que produjeron una especial subjetividad en los subordinados, donde la violencia, el saqueo, el rapto, la tortura se interiorizan como obvias y normales en la Institución. Capellanes, obispos y profesionales formaron parte de esta increíble perversión social. Sabemos que no hay terrorismo de Estado sin complicidad civil, y ésta se vuelve protagónica para que las maquinarias represivas continúen intactas. La complicidad civil también produce mayor represión. El fascismo sigue presente. Los micro fascismos de los pequeños Hitler de Barrio." De aquí que consideramos que estos micro fascismos cotidianos, no sólo están cristalizados en vastos sectores de la institución policial, ya sea provincial, metropolitana o federal, sino que se reproducen expandiéndose hacia otros sectores, sean de la clase social que sean.

Las técnicas de gobernar a los otros y a uno mismo, se han configurado en estos últimos años, teniendo como inoculación preponderante los comportamientos de estos funcionarios. Además de identificaciones con modelos sociales represores, los 
efectos de estos modos de ejercer la libertad con predominancia de la represión (por sobre los de expresión, sobre el de circular libremente, sobre el de manifestarse masivamente, sobre de optar por religión y sexualidad) por parte de las fuerzas de seguridad, han tenido una proliferación micro política, que aún se percibe.

Pero, el hecho de que numerosos grupos de defensores de derechos humanos tengan últimamente viva voz, significa que han iluminado certera y verazmente sobre estos delitos de los cuales antes nada se decía, porque nada se veía, nada se investigaba y nadie era culpable. El trabajo constante de denuncia pública y de movilización permanente, sumada a una profesionalización de sus conocimientos y un reconocimiento simbólico y material de las sociedades civiles internacionales, ha logrado, por fin, tener reconocimiento nacional a nivel gubernamental y, lo más fundamental, tener receptores en los ciudadanos argentinos que se identifican con esta forma de investigar y conocer la vida real en Argentina.

\section{BIBLIOGRAFÍA:}

ABUELAS DE PLAZA DE MAYO; LO GÚGICE, A., (2003) "El lugar del intelectual frente a la vulneración del derecho a la identidad". En "Identidad. Construcción social y subjetiva. Primer Coloquio de Abuelas de plaza de Mayo." Abuelas de Plaza de Mayo. Buenos Aires.

ABUELAS DE PLAZA DE MAYO; CHABABO, R., (2003) "Cómo se construye la identidad." En "Identidad. Construcción social y subjetiva. Primer Coloquio de Abuelas de plaza de Mayo." Abuelas de Plaza de Mayo. Buenos Aires

Comité de Acción Jurídica, (2006) "Situación de los derechos humanos en Argentina. 2004-2006. Jurisprudencia - Comentarios - Debates - Acciones del CAJ." Talleres Trama, Buenos Aires.

CEDAW, (2002) "Derechos Humanos de las mujeres: Asignaturas pendientes del Estado Argentino. Contra informe." Comité CEDAW. 2002.

Centro de Estudios Legales y Sociales (CELS), (2003) "El Estado frente a la protesta social 1996-2002". Temas para pensar la crisis. Siglo veintiuno editores argentina. Buenos Aires, 2003.

- (2005) "Colapso del sistema carcelario. Temas para pensar la crisis". Siglo Veintiuno Editores S. A., Buenos Aires.

- (2005), "Derechos Humanos en Argentina. Informe 2005". Siglo XXI Editores S. A., Bs. As. Argentina.

- (2008), "Derechos Humanos en Argentina. Informe 2010". Siglo XXI Editores S. A., Bs. As. Argentina.

- (2008a) CELS, "Vidas Arrasadas. La segregación de las personas en los asilos psiquiátricos argentinos. Derechos Humanos y salud Mental" Siglo Veintiuno Editores S. A., Buenos Aires.

- (2009) "Derechos Humanos en Argentina. Informe 2010". Siglo XXI Editores S. A., Bs. As. Argentina.

- (2010) "Derechos Humanos en Argentina. Informe 2010". Siglo XXI Editores S. A., Bs. As. Argentina. 
CORREPI, (2006) “Informe de Situación 2003-2006. Los derechos Humanos en la gestión Kirchner”. Bs As.

Equipo Latinoamericano (ELA) (2005): "Informe sobre Género y Derechos Humanos. Vigencia y respeto de los derechos de las mujeres en Argentina". Editorial Biblos. Buenos Aires.

FOUCAULT, M., (1978) "La filosofía analítica de la política”. En Estética, ética y hermenéutica. Editorial Paidós Barcelona, Buenos Aires, México. 1999.

- (1978) "Seguridad, Territorio, Población". Fondo de Cultura Económica de Argentina. S.A. Bs. As., 2006.

- (1979) "Nacimiento De la Biopolítica". Fondo de Cultura Económica, Buenos Aires, 2007.

- (1983a) "El Gobierno de sí y de los otros”. Traducción Ángel Gabilondo. Ediciones Fondo de Cultura Económica.

- (1984) "La ética del cuidado de sí como práctica de la libertad". En Estética, ética y hermenéutica. Editorial Paidós Barcelona, Buenos Aires, México. 1999.

GABILONDO, A., (1999) “La creación de modos de vida”. En Estética, ética y hermenéutica. Editorial Paidós Barcelona, Buenos Aires, México. 1999.

GRANDE A., (2002) "Psicoanálisis Implicado. La marca social en la clínica actual". Topia Editorial. Colección Psicoanálisis, Sociedad y Cultura.

- (2008) "La sexualidad represora". Alfredo Grande Coomp. Topia Editorial. Colección Fichas para el siglo XXI. Buenos Aires.

Organización Internacional de Migraciones (OIM), (2003) "Migración, prostitución y trata de mujeres dominicanas en la Argentina". Organización Internacional para las Migraciones. Oficina Regional Cono Sur. Buenos Aires.

- (2008b) "La trata de personas en argentina, Chile y Uruguay. Estudio exploratorio sobre la trata de personas con fines de explotación sexual en Argentina, Chile y Uruguay”. Organización Internacional para las Migraciones. Misión con Funciones Regionales para el Cono Sur. Buenos Aires. 